\title{
Human Recognition Based on Gait Poses ${ }^{\star}$
}

\author{
Raúl Martín-Félez, Ramón A. Mollineda, and J. Salvador Sánchez \\ Institute of New Imaging Technologies and Dept. Llenguatges i Sistemes Informàtics \\ Universitat Jaume I. Av. Sos Baynat s/n, 12071, Castelló de la Plana (Spain) \\ \{martinr, mollined, sanchez\}@uji.es
}

\begin{abstract}
This paper introduces a new approach for gait analysis based on the Gait Energy Image (GEI). The main idea is to segment the gait cycle into some biomechanical poses, and to compute a particular GEI for each pose. Pose-based GEIs can better represent body parts and dynamics descriptors with respect to the usually blurred depiction provided by a general GEI. Gait classification is carried out by fusing separated posebased decisions. Experiments on human identification prove the benefits of this new approach when compared to the original GEI method.
\end{abstract}

Keywords: gait recognition, GEI, pose estimation, decision level fusion

\section{Introduction}

Gait can be defined as a manner or style of walking. Interestingly, there are studies asserting that every individual has a unique gait pattern [3], what has lead gait to be considered as a new biometric feature. When compared to other biometric features such as face, voice or fingerprint, gait has several attractive properties. It can be reliably perceived at a greater distance with simple instrumentation, and it does not require the cooperation or awareness of the individual.

There exist many applications that could benefit from gait analysis, including surveillance, diagnosis and treatment of gait-related disorders, motion capture in computer graphics and games, and so on.

However, there are also several factors that hinder the use of gait as a biometric feature. For instance, gait analysis is very sensitive to segmentation of the subject's silhouette, but also footwear, clothing, carrying conditions and walking speed may affect gait by reducing its discriminative power as a biometric. Even so, it is still useful to complement other biometric features under certain conditions (e.g. uncooperative subject, low quality images, etc.).

In literature, two main approaches have been proposed to obtain gait patterns from video sequences [1]: model-based and model-free methods. Proposals in the first group aim at recovering a structural model of human motion $[8,11]$ by

\footnotetext{
* Partially supported by projects CSD2007-00018 and CICYT TIN2009-14205-C04-04 from the Spanish Ministry of Innovation and Science, P1-1B2009-04 from Fundació Bancaixa and PREDOC/2008/04 grant from Universitat Jaume I. Portions of the research in this paper use the CASIA Gait Database collected by Institute of Automation, Chinese Academy of Sciences.
} 
matching the joint locations with a robust kinematic model of the human body. However, this is a hard task because of some problems such as occlusion of body parts, joint angle singularities, etc. On the other hand, model-free methods $[4,7]$ do not use any model, and they are based on changes of the subject's appearance, which implicitly contain information about body movements.

Probably, the best-known model-free method is gait energy image (GEI) [4], which obtains an average silhouette image to represent both body shape and movements over a gait cycle. Although several potentially discriminative body parts usually appear blurred in GEI images (chest and back regions caused by movements of arms, and shape of arms and legs because of their motion), this method has proved to be effective in many tasks, such as human identification [13] and gender classification [12].

Biomechanical studies [9] assert that several recognizable poses happen successively in a gait cycle, e.g., those in which legs are spread, legs are closest together, etc. A first attempt to segment recognizable poses from gait video sequences was carried out by selecting a set of key frames representing some poses [2], but results on a human recognition task mostly depended on quality of the chosen frames.

In this work, a new way of using GEI is proposed and its effectiveness is compared with that of the original method. The main hypothesis supporting the proposal is that shape of body parts could be better noticed if different GEIs were separately obtained for some predefined key poses within a gait cycle [9]. Furthermore, they could also provide a few dynamic descriptors usually blurred in original GEI. By following this idea, several silhouettes are used to represent each pose instead of using a unique key frame as in [2], what is expected to be more robust to noise from individual frames. In the classification stage, each pose-based GEI is individually classified, and these decisions are fused to produce the subject recognition. Experiments prove the higher performance of this

combined solution with respect to results obtained from the original GEI and from the particular key poses.

\section{Background}

This section describes the original GEI method and gives some information about the biomechanical phases and poses involved in a gait cycle.

\subsection{Gait Energy Image (GEI)}

As already said, GEI basically generates an average silhouette for a gait cycle, which reflects shape of the body parts and in some extent, their changes over time (gait dynamics). In this way, it reduces storage and time requirements and it is also more robust to noise of individual frames.

Before computing the GEI of a given gait sequence, its frames must be preprocessed as follows: 
- Foreground segmentation. Given a frame, the aim is to obtain an image in which foreground is segmented from background and a silhouette is highlighted. It could be simply done, for instance, by background subtraction.

- Silhouette extraction. From each foreground frame, a cropped image is extracted from the bounding box that encloses all silhouette pixels.

- Size normalization and horizontal alignment. The silhouette image is scaled to a new one having a pre-fixed common height and a variable width to keep its original aspect ratio. Then this normalized silhouette is horizontally centered in a template of fixed sizes from the horizontal centroid value of its upper-half, since this part of the body involves fewer changes than the lower-half when a person walks.

Afterwards, given the set of preprocessed silhouettes of a gait video sequence $\left\{I_{t}(x, y)\right\}$ with $1 \leq t \leq N, N$ being the number of silhouettes, and $(x, y)$ referring to a specific position in the 2D image space, each gray-level pixel of a GEI is computed as $G E I(x, y)=\frac{1}{N} \sum_{t=1}^{N} I_{t}(x, y)$.

\subsection{Phases and Key Poses Within a Gait Cycle}

According to biomechanical studies [9], walking is a cyclic process of limb motion to move the body forward while balance is simultaneously kept. Therefore, the relevant information of a gait pattern can be captured from a whole gait cycle (or stride), which is the interval between two sequential foot contacts by the same limb.

Limbs goes through two phases while walking. The first one is Double Limb Stance. It comprises the period of time in which both feet are on the ground for the transfer of body weight from the support limb to the other. The second phase is Single Limb Stance that consists of a larger period of time after the first phase in which one limb serves as a mobile source of support while the other limb is advancing or swinging to a new support place. Then limbs interchange their roles and a new two-phase succession completes the gait cycle.

More specifically, eight main poses can be distinguished during a gait cycle when both limbs are separately considered, but they can be reduced to four poses if the expected symmetry of both halves of a gait cycle is taken into account.

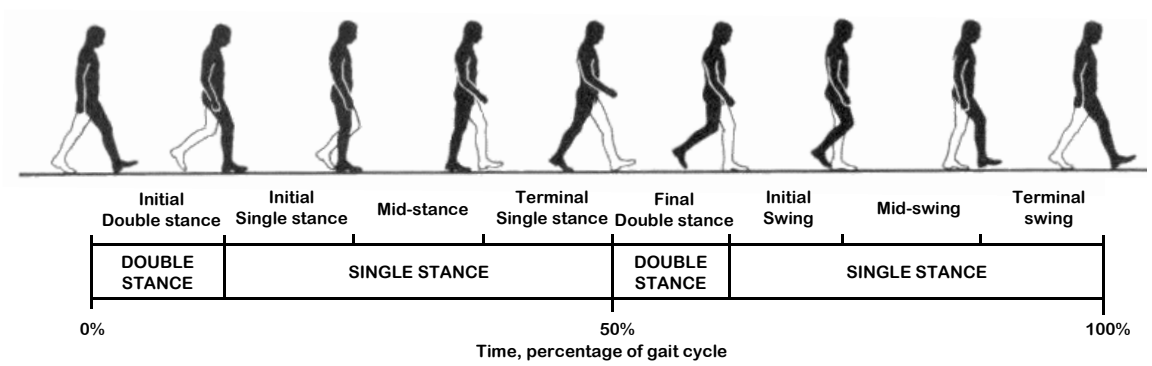

Fig. 1. Phases and poses within the gait cycle. 


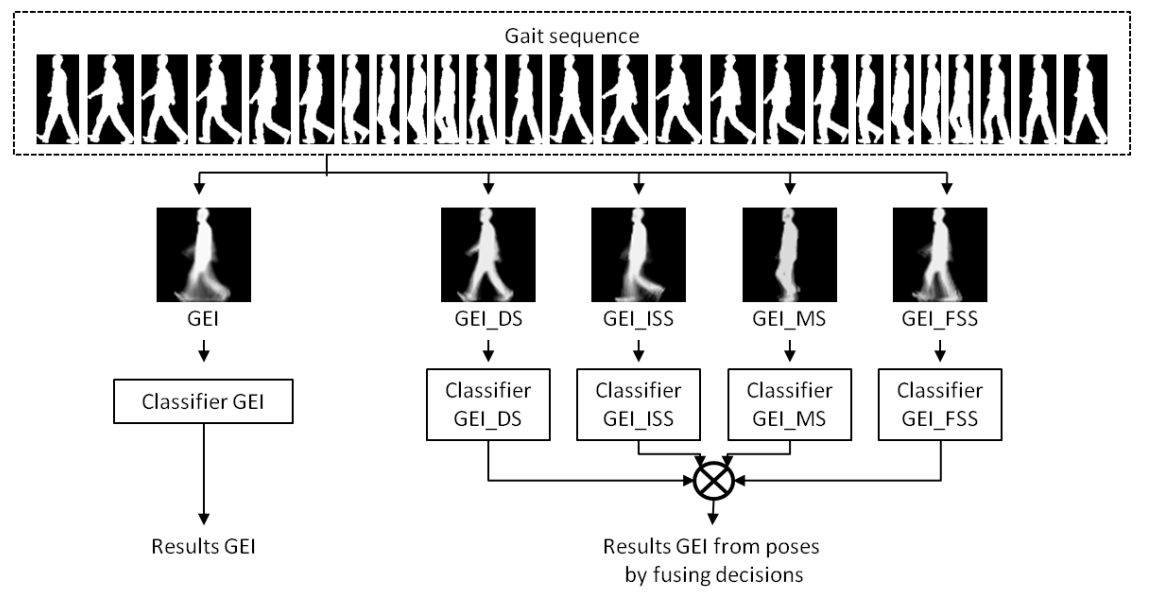

Fig. 2. General solution scheme.

Figure 1 illustrates a full gait cycle showing the eight poses and the different phases.

\section{Methodology}

This section provides the basis of the method here proposed along with the details to generate GEIs for the different poses.

\subsection{Fundamentals of the Proposed Method}

The new way of using GEI here proposed is based on three main assumptions:

Assumption 1. Focusing on Figure 1, poses of the two halves of a gait cycle are expected to be symmetric if information about what limb is closer to the camera is unavailable. Therefore the step could be chosen as the gait cycle measure instead of the stride (unlike most of related works $[4,13,12]$ ).

Assumption 2. By using several pose-based GEIs, each one associated to a particular key pose, the shape of all body parts could be reflected in a more accurate way than in the representation given by a unique GEI averaging all poses. In addition, they could better show some dynamic features such as the length of the stride and extent of arm swing.

Assumption 3. Given a gait sequence with multiple gait cycles, most of works compute a different GEI for each gait cycle. However, a better representation could be obtained by comprising all cycles in a unique GEI, since noise of individual silhouettes could not affect so much, and it could lead to more efficient algorithms. If assumptions 2 and 3 are satisfied, pose-based GEIs could be generated from a higher number of frames. 


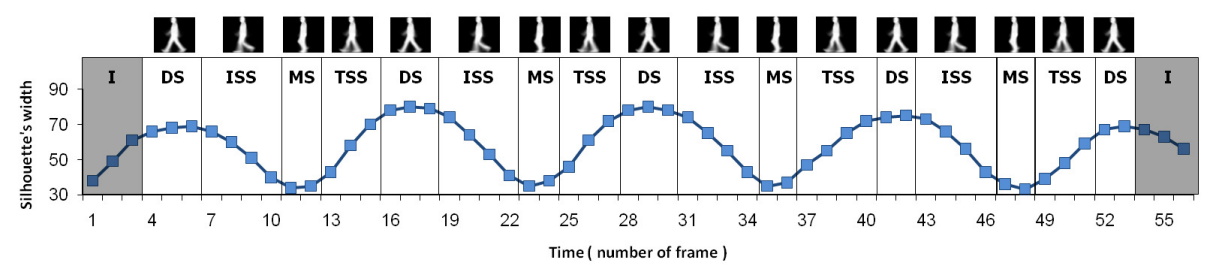

Fig. 3. Classifying frames in poses by their width.

Under these assumptions, given a test gait sequence, the plain GEI of the whole sequence (image called GEI in Figure 2) and four pose-based GEIs during the complete sequence (images called GEI_DS, GEI_ISS, GEI_MS and GEI_FSS in Figure 2) are computed.

A general overview of the method, which has been illustrated in Figure 2, is now introduced. Firstly, individual frames are classified in one of four poses following the procedure detailed in Section 3.2. Then, for the whole sequence, all silhouettes belonging to a given pose are averaged to compute its corresponding GEI. From each one of the four pose-based GEIs, an individual decision about the identity of the subject is given according to the label of the most similar training GEI among those of the same pose. Finally, these four decisions are fused by majority voting to produce a unique decision more robust and probably more reliable than the individual decision obtained from the plain use of GEI. In case of a tie, the system rejects the classification of the corresponding sample.

\subsection{Creating GEIs of Each Pose}

In order to classify each silhouette in a landmark pose, the periodic signal provided by the silhouette width as a function of time for the whole sequence is used in a similar way to the work by Collins et al. [2]. As can be seen in Figure 3, the sequence of side-view silhouettes of a person walking defines a periodic function with peaks and valleys. The silhouette width alternatively expands (peaks) and contracts (valleys) over time as the person's legs spread and come back together again during the gait cycle. Therefore, each step comprises from peak to peak in the periodic signal.

It conducts to the following classification of frames into gait poses:

1. Double limb Stance (DS), the frame at the current peak and those frames in the neighbourhood with a very close width value (both legs spread and touching the ground).

2. Initial Single Stance (ISS), from the last frame of DS to a frame that surrounds the next valley (the front leg is on the ground and the rear leg is swinging towards it).

3. Mid-Stance (MS), the frame at the current valley and those frames in the neighbourhood with a very close width value (legs are closest together with the swinging leg just passing the static one). 
4. Terminal Single Stance (TSS), from the last frame of MS to a frame at the surroundings of the following peak (the supporting leg is now the rear one, and the swinging leg appears as the front leg).

This simple and robust method is able to accurately determine to which pose each frame belongs to. Figure 3 shows an example of how silhouettes are labelled.

\section{Experiments and Results}

Experiments aim at assessing the effectiveness of combining pose-based decisions with respect to a plain classification using the original GEI method.

\subsection{Database Description and Preprocessing}

Experiments are carried out on CASIA Gait Database [5] - Database B, which consists of videos from 124 subjects. Only the six gait sequences in which each subject appears walking in their side-view are considered, what gives a total of 744 sequences to be used in experiments. In addition, this database provides well-segmented foreground images that have been used in this work as inputs to the silhouette extraction step (see Section 2.1).

Once silhouettes have been obtained, they are scaled and horizontally aligned to images with two different prefixed sizes (32x32 and $64 \times 64$ pixels) in order to measure the effect of image size. The gray-level value of each pixel is considered a different feature, thus a high dimensionality should be managed (more than 1000 features). In order to avoid this problem, dimensionality is reduced by using the well-known PCA technique [6]. In this way, the original GEI features are projected onto a smaller number of new uncorrelated ones that accounts for a given percentage of the variance ( $95 \%$ in this work).

\section{$4.2 \quad$ Experimental Setup}

Apart from the main objective previously described, experiments also aim at measuring effects of dimensionality reduction with PCA. Due to the limited number of samples available in the database (744), the leaving-one-out method is used to estimate the performance of each strategy. Since the CASIA Database contains several gait sequences for each subject, at least one gait sequence belonging to the tested subject is in the gallery set.

The nearest neighbor classifier (1NN) was here selected because of its simplicity and common good performance. With respect to the distance metric, the Euclidean distance was used for experiments without PCA because all original attributes share the same domain, but the Mahalanobis distance was used when PCA was applied since it is able to remove the dominance of features with large variance in the transformed space. 


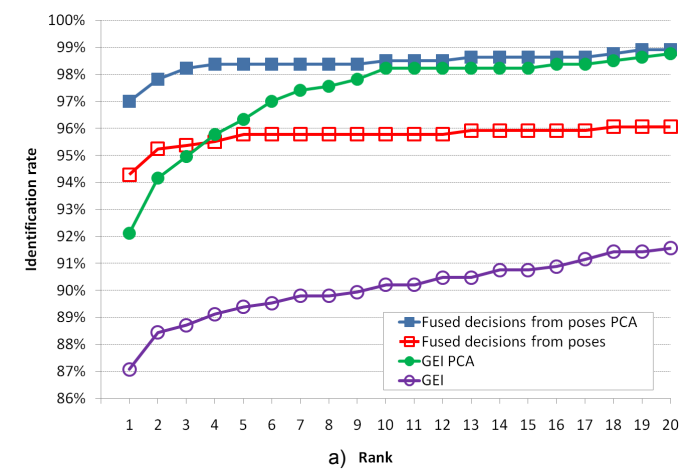

a) Rank

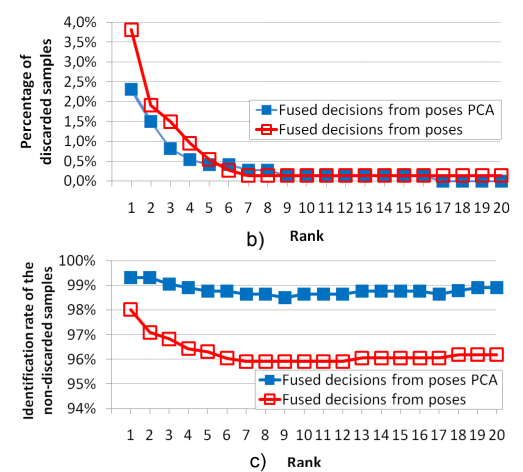

c) Rank

Fig. 4. a) Comparison of the methods with CMS. b) Percentage of discarded samples of the new method. c) CMS over non-discarded samples with the new method.

\subsection{Analysis of Results}

In this work, performance is assessed by Cumulative Match Scores (as in the FERET scheme [10]) and it is shown in Figure 4 a). This chart depicts the percentage of test sequences whose same-class nearest neighbour is among the top x matches. In this way, Rank 1 value is the classification correct rate $(\mathrm{CCR})$, i.e., the percentage of subjects correctly classified at the first match.

Figure 4 a) reflects results of both aims of this work. It compares the performance of the new method with that of the usual GEI and, at the same time, it shows the effect of reducing dimensionality by PCA in both methods. It is very important to remark that results of the new method are considering the worst case (all ties are counted as errors). By analysing this figure, a first conclusion is that the new method performs better than the original GEI. It can be easily seen by comparing their CCRs (Rank 1). They are $97 \%$ and $94 \%$ for the new method using PCA or not respectively, and $92 \%$ and $87 \%$ in the same cases for the original GEI method. Such experimental results are probably supported by the fact that the combination of heterogeneous decisions from different poses provides more and better information than the original method. Finally, another conclusion is that both methods benefit from the use of PCA, not only in terms of performance improvement (3-7\% higher for all ranks in both methods), but also in terms of reducing the computational complexity and dimensionality.

Figure $4 \mathrm{~b}$ ) shows the percentage of samples rejected due to ties, while the Figure $4 \mathrm{c}$ ) depicts the identification rate over the non-discarded samples. From the analysis of these two charts together, very encouraging results are found. As the rank increases, the number of discarded samples (ties) drops drastically, while the success rate of the new method using PCA keeps almost steady and very close to $99 \%$. It means that if the low percentage of ties is accepted (lower than $2.5 \%$ ), the identification rate increases from about $97 \%$ (when ties are counted as errors, see Figure 4 a)) to about the $99 \%$ previously commented. When PCA is not applied, the classifier performance is slightly affected. 
Results of methods using GEIs of size $64 \times 64$ are not shown in this paper because they follow a similar trend and they are more computationally demanding.

\section{Conclusion}

This paper proposes a new method for characterizing key poses of the gait pattern by individual GEIs. These pose-based representations implicitly capture biometric shape features (e.g. body part proportions) and dynamic descriptors (e.g. amount of arm swing) more accurately than usual GEI. Classification results obtained from combining individual pose-based decisions were better than those from the plain use of the original GEI method for the whole gait sequence. Besides, the new method is robust to noisy data and it is easy to understand.

However, it suffers from view dependence and it is limited to classify test sequences taken from roughly the same viewing angle as the training sequences. Our future research could be addressed to extend this method to mitigate the impact of such a problem.

\section{References}

1. Boulgouris, N., Hatzinakos, D., Plataniotis, K.: Gait recognition: a challenging signal processing technology for biometric identification. IEEE Signal Processing Mag. 22(6), 78-90 (2005)

2. Collins, R.T., Gross, R., Shi, J.: Silhouette-based human identification from body shape and gait. In: Proc. IEEE Int'l. Conf. Automatic Face and Gesture Recognition. pp. 366-371 (2002)

3. Cutting, J., Kozlowski, L.: Recognizing friends by their walk: Gait perception without familiarity cues. Bull. Psychonomic Soc. 9(5), 353-356 (1977)

4. Han, J., Bhanu, B.: Individual recognition using gait energy image. IEEE Trans. Pattern Anal. Mach. Intell. 28(2), 316-322 (2006)

5. Institute of Automation, Chinese Academy of Sciences: CASIA Gait Database (2005), http://www.sinobiometrics.com

6. Jolliffe, I.: Principal Component Analysis. Springer, second edn. (2002)

7. Kale, A., Rajagopalan, A.N., Sundaresan, A., Cuntoor, N., Roychowdhury, A., Krueger, V.: Identification of humans using gait. IEEE Trans. Image Process. 13(9), $1163-1173$ (2004)

8. Lee, L., Grimson, W.: Gait analysis for recognition and classification. In: Proc. 5th IEEE Int'l. Conf. Automatic Face and Gesture Recognition. pp. 155-162 (2002)

9. Perry, J.: Gait Analysis: Normal and Pathological Function. SLACK Inc. (1992)

10. Phillips, P., Moon, H., Rizvi, S., Rauss, P.: The feret evaluation methodology for face-recognition algorithms. IEEE Trans. Pattern Anal. Mach. Intell. 22(10), 10901104 (2000)

11. Yam, C., Nixon, M., Carter, J.: Automated person recognition by walking and running via model-based approaches. Pattern Recognit. 37(5), 1057-1072 (2004)

12. Yu, S., Tan, T., Huang, K., Jia, K., Wu, X.: A study on gait-based gender classification. IEEE Trans. Image Process. 18(8), 1905-1910 (2009)

13. Yu, S., Tan, D., Tan, T.: A framework for evaluating the effect of view angle, clothing and carrying condition on gait recognition. In: Proc. 18th Int'l. Conf. Pattern Recognition. pp. 441-444 (2006) 